\title{
A Novel Scoring System to Correlate Preoperative Adrenal Imaging with Final Histology in Predicting Malignancy: A Retrospective Institutional Data Analysis
}

\author{
Loreno Enny ${ }^{1}$, Kushagra Gourav ${ }^{2}$, Shruti Singh ${ }^{3}$, Pooja Ramakant ${ }^{4}$, Kul Ranjan Singh ${ }^{5}$, Anand Mishra ${ }^{6}$
}

\begin{abstract}
Background and aim: To investigate whether preoperative contrast-enhanced computed tomography (CECT) will be able to differentiate benign adrenal mass from malignant mass by applying a combination of morphological criteria and correlating it with the final histopathology. Materials and methods: Thirty-six patients planned for surgery were assessed preoperatively with CECT abdomen and based on CT findings a score of 0 to 6 was given depending on the size of the lesion, margin, density, abutment/infiltration to surrounding structures, necrosis, and calcification which was further correlated with their final histopathology.

Statistical test used: The reliability of the total score was checked using receiver operator characteristic (ROC) analysis.

Results: Out of 36 patients, 28 were benign and 8 were malignant pathology. The presence of abutment, ill-defined margins, and necrosis on CT was found significantly more in patients with malignant pathology $(p=0.009, p=0.005$, and $p=0.009$, respectively). The most sensitive and specific parameters in predicting malignancy based on CT were heterogeneity and ill-defined margins, respectively (100 and $94.6 \%$, respectively). The cut-off score of four has a sensitivity, specificity, NPV, and PPV of 75, 82.1, 54.55, and 92\%, respectively. The mean scores for benign and malignant were $2.21 \pm 0.315$ and $4.25 \pm 1.581$, respectively $(p=0.001)$.

Conclusion: This novel scoring method including heterogeneity, margins, abutment, and necrosis plays a key role in predicting malignancy accurately on CT scans.

Keywords: Adrenal mass, Adrenocortical carcinoma, Adrenal Surgery, Contrast-enhanced computed tomography.

World Journal of Endocrine Surgery (2020): 10.5005/jp-journals-10002-1310
\end{abstract}

\section{INTRODUCTION}

Adrenal tumors have always been under the horizon of surgeon's fascinations. Being suprarenal in location, and surrounded by many vital structures like kidney, liver, and inferior vena cava; surgical planning of adrenal tumors has always been a challenge. Successful adrenal surgery often demands a prior accurate clinical diagnosis and road map to plan the surgical approach and anticipate intraoperative difficulties.

Adrenal gland lesions are seen with a wide range of clinical scenarios due to the variety of hormones secreted by the glands. Expert knowledge of such classical clinical presentations and their pathological hormonal milieu associated with various adrenal gland neoplasms gives an extra edge to endocrine surgeons. Mostly adrenal gland neoplasms secrete hormones and are thus overtly functional (e.g., secondary hypertension in pheochromocytoma, Cushing's syndrome, Conn's adenoma), whereas, on the other end of the spectrum, non-functional adrenal masses are usually discovered otherwise as incidentalomas (adenoma, metastases).

Clinical manifestations of hormonal hypersecretion like hypertension, Cushingnoid habitus - a classical feature of many benign adrenal lesions such as pheochromocytoma and Cushing's adenoma, respectively; may also be seen in malignant pheochromocytoma or adrenocortical carcinoma (ACC). The non-functional adrenal adenomas constitute about $75 \%$ of adrenal incidentalomas, a high proportion (40-60\%) reflects metastatic disease in the oncological population. ${ }^{1-3}$ Prognosis and management depend on the presence or absence of malignancy.

Interpretation of findings on contrast-enhanced computed tomography (CECT) and MRI abdomen are areas of focus as a
${ }^{1,4-6}$ Department of Endocrine Surgery, King George's Medical University, Lucknow, Uttar Pradesh, India

${ }^{2}$ Department of General Surgery, King George's Medical University, Lucknow, Uttar Pradesh, India

${ }^{3}$ Department of Community Medicine and Public Health, King George's Medical University, Lucknow, Uttar Pradesh, India

Corresponding Author: Pooja Ramakant, Department of Endocrine Surgery, King George's Medical University, Lucknow, Uttar Pradesh, India, Phone: +91 9791507780, e-mail: poojaramakant@gmail.com

How to cite this article: Enny L, Gourav K, Singh S, et al. A Novel Scoring System to Correlate Preoperative Adrenal Imaging with Final Histology in Predicting Malignancy: A Retrospective Institutional Data Analysis. World J Endoc Surg 2020;12(3):122-127.

Source of support: Nil

Conflict of interest: None

roadmap in surgical planning among surgeons. Though scientists across the globe are working hard to devise modalities to preoperatively detect/suspect malignancy in adrenal lesions; still it is very difficult to confirm malignancy on radiology criteria alone. In the last 25 years, the imaging and treatment of adrenal gland pathology have been the focus of intensive study and controversy along with the use of cross-sectional imaging modalities as a key component of clinical care. To prevent more costly tests (FDG-PET) or invasive procedures (biopsies), as many patients as possible must be diagnosed on findings of the CT adrenal mass. Therefore, we aim to investigate whether preoperative CECT will be able to

(c) Jaypee Brothers Medical Publishers. 2020 Open Access This article is distributed under the terms of the Creative Commons Attribution 4.0 International License (https://creativecommons.org/licenses/by-nc/4.0/), which permits unrestricted use, distribution, and non-commercial reproduction in any medium, provided you give appropriate credit to the original author(s) and the source, provide a link to the Creative Commons license, and indicate if changes were made. The Creative Commons Public Domain Dedication waiver (http://creativecommons.org/publicdomain/zero/1.0/) applies to the data made available in this article, unless otherwise stated. 
differentiate benign adrenal mass from the malignant mass by applying a combination of morphological criteria and correlating it with the final histopathology.

\section{Study Methods}

This is a retrospective study and the patient data were collected from the hospital records in the Departments of Endocrine Surgery and Pathology, King George Medical University Lucknow, India. The study period included all patients with adrenal masses planned for surgery between January 2017 and March 2020.

Parameters including patient's demographic status, clinical presentation, preoperative imaging including CECT abdomen, hormonal work-up, definitive treatment, and the final histopathological result were collected from medical records.

All patients planned for surgery were assessed preoperatively with CECT and on the basis of CT finding, a score of 0 to 6 was given depending on the size of the lesion $(0=<6 \mathrm{~cm}, 1=\geq 6 \mathrm{~cm})$, margin $(0=$ well-defined, $1=$ ill-defined $)$, density $(0=$ homogeneous, $1=$ heterogeneous), abutment/infiltration to surrounding structures ( $0=$ no abutment, $1=$ abutment $)$, necrosis $(0=$ no necrosis, $1=$ presence of necrosis), and calcification ( $0=$ no calcification, $1=$ presence of calcification) (Figs 1and 2). The added score (0-6) was then correlated with the final histopathology. Hypothesis was that higher the score, higher the chance of malignancy.

\section{Statistical Analysis}

Data were analyzed and reported as the mean \pm SD for continuous variables and frequency (percentage) for categorical variables. The statistical analysis was performed using an unpaired $t$-test. Significance was set at $p$ value $<0.05$ and receiver operator characteristic (ROC) curve was used to check sensitivity, specificity, positive predictive value (PPV), and negative predictive value (NPV) of each cut-off score.

\section{Results}

The final study population consisted of 36 patients with a female to male ratio of 1.6:1. There were $28(77.8 \%)$ benign and $8(22.2 \%)$ malignant pathology (Table 1). The mean age of the study population was $33.4 \pm 14.9$ years. The mean size of the tumor on CT for the entire cohort was $6.5 \pm 2.7 \mathrm{~cm}$ (range from 1.6 to $15 \mathrm{~cm}$ ) (91.7\%) (Table 1).

Open adrenalectomy was the most common surgery performed in 22 patients (61.1\%). Laparoscopic and retroperitoneoscopic adrenalectomy was performed in $5(11.1 \%)$ and 5 (11.1\%), respectively. The most common indication for surgery was benign pheochromocytoma, 9 (25\%) followed by adrenal myelolipoma in 7 patients (19.4\%). Adrenocortical carcinoma was the most common malignant pathology, $3(8.3 \%)$ followed by malignant pheochromocytoma in 2 patients (5.6\%) (Table 1).

The presence of abutment to the surrounding structure, illdefined margins, and necrosis on CECT was found significantly more in patients with malignant adrenal mass as compared to those with benign lesion but no significant difference was found based on the size of the lesion, density, and margins (Table 2).

The presence of abutment on CT had a sensitivity, specificity, NPV, and PPV of 75, 78.6, 50, and 91.7\%, respectively, presence of calcification had a sensitivity of only $37.5 \%$ and specificity of $67.9 \%$, size of lesion $>6 \mathrm{~cm}$ was $75 \%$ sensitive and $50 \%$ specific heterogeneity on CT had sensitivity and specificity of 100 and $17.9 \%$, respectively, presence of ill-defined margins had a sensitivity of $50 \%$ and specificity of $96.4 \%$ and presence of necrosis was $75 \%$ sensitive and $78.6 \%$ specific in predicting malignancy. The most sensitive parameter in predicting malignancy based on CT findings
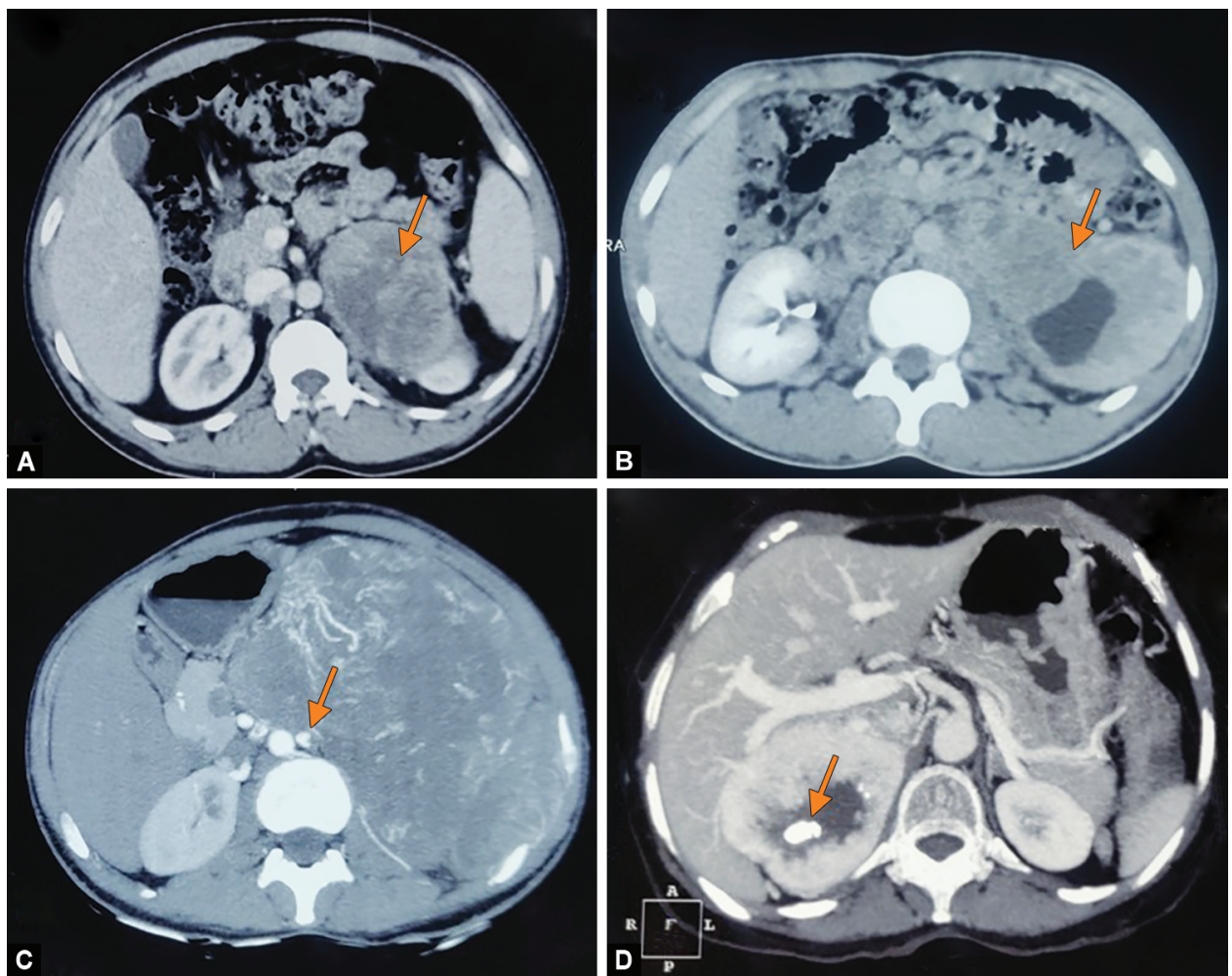

Figs 1 A to D: (A) Left heterogeneous adrenal lesion (arrow); (B) Left adrenal lesion with ill-defined margins (arrow); (C) Large adrenal lesion abutting surrounding structures with loss of fat planes (arrow); (D) Right adrenal lesion with presence of calcification within the lesion (arrow) 

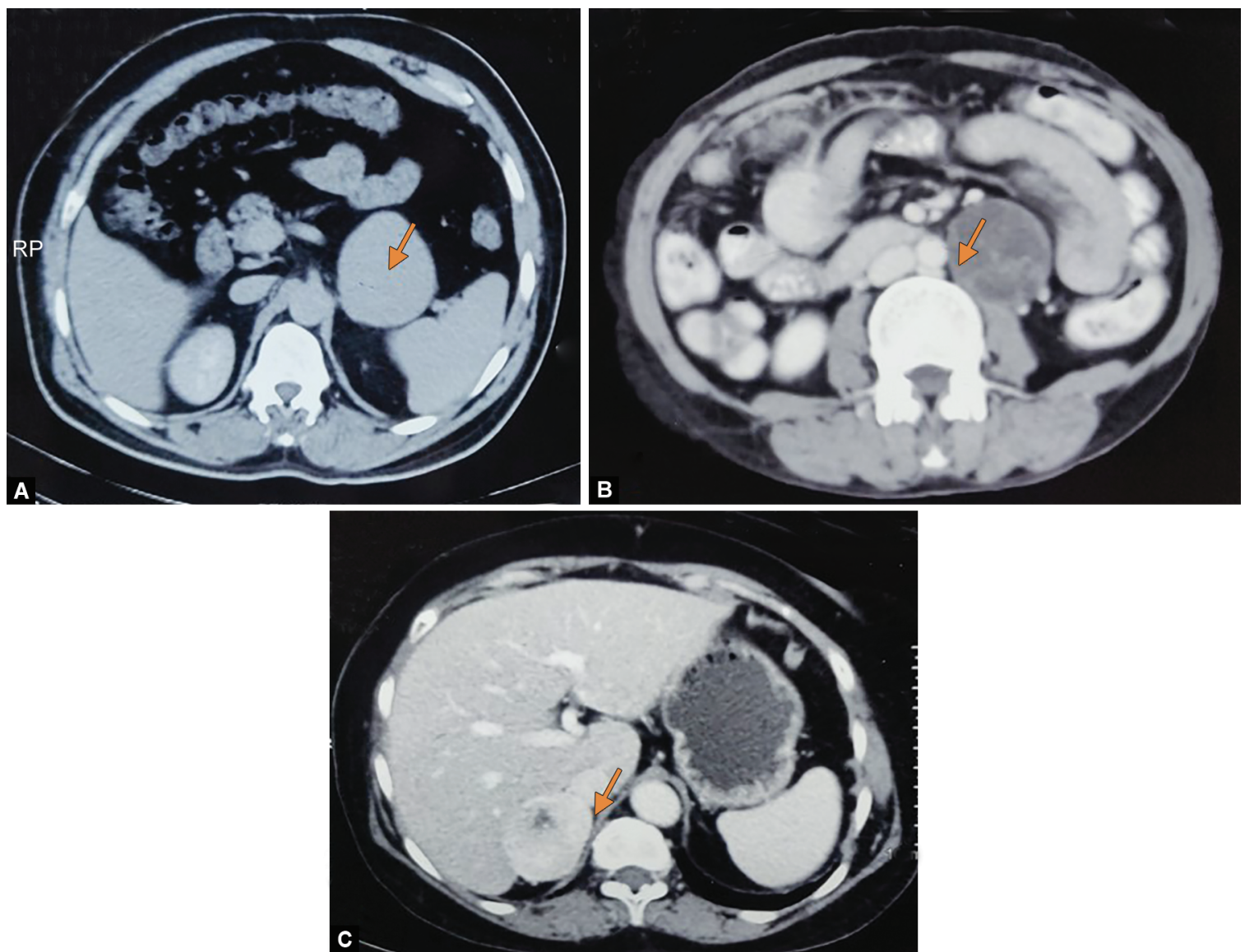

Figs 2A to C: (A) Left homogeneous adrenal lesion (arrow); (B) Right adrenal lesion with free from surrounding structure (arrow); (C) Right adrenal lesion with well-defined margins (arrow)

was heterogeneity of the lesion ( $100 \%$ sensitive) whereas the most specific parameter was the presence of ill-defined margins on $\mathrm{CT}$ (96.4\% specific) (Table 3).

Based on ROC analysis, a cut-off score of 4 was taken with a sensitivity, specificity, NPV, and PPV of 75, 82.1, 54.55, and 92\%, respectively (Fig. 3). Table 4 shows the sensitivity, specificity, PPV, and NPV of each cut-off score. The mean score for benign pathology was $2.21 \pm 0.315$ and that of the malignant pathology was $4.25 \pm$ 1.581 (Table 5).

\section{Discussion}

Though different morphological features of adrenal CT imaging have been studied extensively in different adrenal pathologies; most of them are performed on "Unenhanced CT", still there is very limited data over scoring criteria that accurately distinguish between benign and malignant adrenal masses on CECT imaging. ${ }^{4}$

Our analysis aimed to test six different morphological parameters of CECT (size, margins, abutment, necrosis, calcification, and homogeneity) to improve the accuracy of the diagnosis. We found four parameters heterogeneity, margins, abutment, and necrosis as the most distinct features differentiating malignant adrenal masses from benign ones.

Sensitivity and specificity values were calculated against multiple cut-off values for this score. As the higher cut-off values were considered sensitivity was decreasing and specificity values were in increasing trend. So, a cut-off value of $\geq 4$ was considered with reasonable high values for sensitivity, specificity, and NPV
(Table 5). Keeping the cut-off value of 4 was also supported by the fact that the mean score for malignant adrenal masses was $4.25 \pm 1.581$ and there was a statistically significant difference $(p$ value-0.001) between mean scores for benign masses $(2.21 \pm 0.315)$ and malignant masses $(4.25 \pm 1.581)$ (Table 5).

Gufler et al. described similar scoring criteria to differentiate among adenoma and adrenal metastasis that combined four parameters - size, contour, homogeneity, and density. ${ }^{5}$ As a contrasting point from our study, Gufler et al. analyzed unenhanced CT scans of 56 patients with an adrenal mass and a history of an extra-adrenal malignancy-24 were adenomas and 32 were found to be metastases. ${ }^{5}$ So, their model was altogether different from ours where we studied only primary benign and malignant masses of adrenal. Among other models, PET is extremely productive in differentiating benign and malignant adrenal lesions with sensitivity and specificity of 94 and $100 \%$, respectively. ${ }^{6}$

While considering individual imaging parameters, the size of the adrenal lesion has been one of the most unique criteria of all. Many adrenal incidentaloma treatment guidelines are still focused on the size of the adrenal mass. ${ }^{7-9}$ The size has some prescient worth; be that as it may, as most adenomas are under $4 \mathrm{~cm}$, while ACCs have a median size of $11 \mathrm{~cm} .{ }^{10}$ Quite contrasting with this; mean tumor size on CT was $6.5 \pm 2.7 \mathrm{~cm}$ in our study. Solely taking a cut-off of $6 \mathrm{~cm}$ would miss $25 \%$ of our ACC lesions. This may be because on taking $6 \mathrm{~cm}$ as the cut-off for scoring, only 6 out of 8 malignant adrenal masses (75\%) had size $>6 \mathrm{~cm}$; while benign adrenal masses were equally distributed on either side of the $6 \mathrm{~cm}$ 
Table 1: Patients demographic characteristics

\begin{tabular}{|c|c|c|}
\hline \multirow[b]{2}{*}{ Parameters } & \multicolumn{2}{|c|}{ Result } \\
\hline & No. & $\%$ \\
\hline Total no. of patients & 36 & \\
\hline \multicolumn{3}{|l|}{ Gender (ratio 1.6:1) } \\
\hline Female & 22 & 61.1 \\
\hline Male & 14 & 38.9 \\
\hline Age (years) & 33.4 & \\
\hline \multicolumn{3}{|l|}{ Laterality } \\
\hline Right & 25 & 69.4 \\
\hline Left & 9 & 25 \\
\hline Bilateral & 2 & 5.6 \\
\hline \multicolumn{3}{|l|}{ Presenting symptoms } \\
\hline No symptoms & 4 & 11.1 \\
\hline Pain in abdomen & 25 & 69.4 \\
\hline Classical triad of pheochromocytoma (hypertension, palpitation, sweating) & 7 & 58.3 \\
\hline Hypertension & 11 & 91.7 \\
\hline Mean size of tumor on CT & $6.5=$ & \\
\hline Mean size of the tumor on HPE & $7.6=$ & \\
\hline \multicolumn{3}{|l|}{ Surgery } \\
\hline Open adrenalectomy & 22 & 61.1 \\
\hline Laparoscopic adrenalectomy & 5 & 13.9 \\
\hline Retroperitoneoscopic adrenalectomy & 5 & 13.9 \\
\hline Lap. converted to open & 4 & 11.1 \\
\hline \multicolumn{3}{|l|}{ Histopathology } \\
\hline Benign & 28 & 77.8 \\
\hline Pheochromocytoma & 9 & 25 \\
\hline Myelolipoma & 7 & 19.4 \\
\hline Adrenal cyst & 4 & 11.1 \\
\hline Paraganglioma & 4 & 11.1 \\
\hline Ganglioneuroma & 2 & 5.6 \\
\hline Conn's adenoma & 2 & 5.6 \\
\hline Malignant & 8 & 22.2 \\
\hline Adrenocortical carcinoma & 3 & 8.3 \\
\hline Pheochromocytoma & 2 & 5.6 \\
\hline Neuroblastoma & 1 & 2.8 \\
\hline Small round cell tumor & 1 & 2.8 \\
\hline Composite tumor & 1 & 2.8 \\
\hline Malignant pheochromocytoma + ganglioneuroma & 1 & 2.8 \\
\hline
\end{tabular}

Table 2: Difference between benign and malignant based on CECT findings

\begin{tabular}{lllcl}
\hline Parameters & & Malignant & Benign & pvalue \\
\hline Abutment & Present & 6 & 6 & 0.009 \\
& Absent & 2 & 22 & \\
Calcification & Present & 3 & 9 & 1.000 \\
& Absent & 5 & 19 & \\
Size of lesion & $\geq 6 \mathrm{~cm}$ & 6 & 14 & 0.257 \\
& $<6 \mathrm{~cm}$ & 2 & 14 & \\
Density & Heterogeneous & 8 & 23 & 0.566 \\
& Homogeneous & 0 & 5 & \\
Margins & III-defined & 4 & 1 & 0.005 \\
& Well-defined & 4 & 27 & \\
Necrosis & Present & 6 & 6 & 0.009 \\
& Absent & 2 & 22 & \\
\hline
\end{tabular}

Table 3: Sensitivity, specificity, NPV, and PPV of each finding on CECT

\begin{tabular}{lcllc}
\hline Parameters & $\begin{array}{l}\text { Sensitivity } \\
(\%)\end{array}$ & $\begin{array}{l}\text { Specificity } \\
(\%)\end{array}$ & PPV (\%) & NPV (\%) \\
\hline Abutment & 75 & 78.6 & 50 & 91.7 \\
Calcification & 37.5 & 67.9 & 25 & 79.2 \\
Size of lesion $(\geq 6 \mathrm{~cm})$ & 75 & 50 & 30 & 87.5 \\
Heterogeneous & 100 & 17.9 & 25.8 & 100 \\
Margins & 50 & 96.4 & 80 & 87.1 \\
Necrosis & 75 & 78.6 & 50 & 91.7 \\
\hline
\end{tabular}

cut-off mark. Hence, the difference among both pathologies was not significant. Considering size alone as a single parameter and 6 $\mathrm{cm}$ as a cut-off, increases our sensitivity (75\%) and NPV (87.5\%) to a fairly good level. Since almost half of the benign adrenal masses enrolled are non-functional — that may be a reason for the delay in 


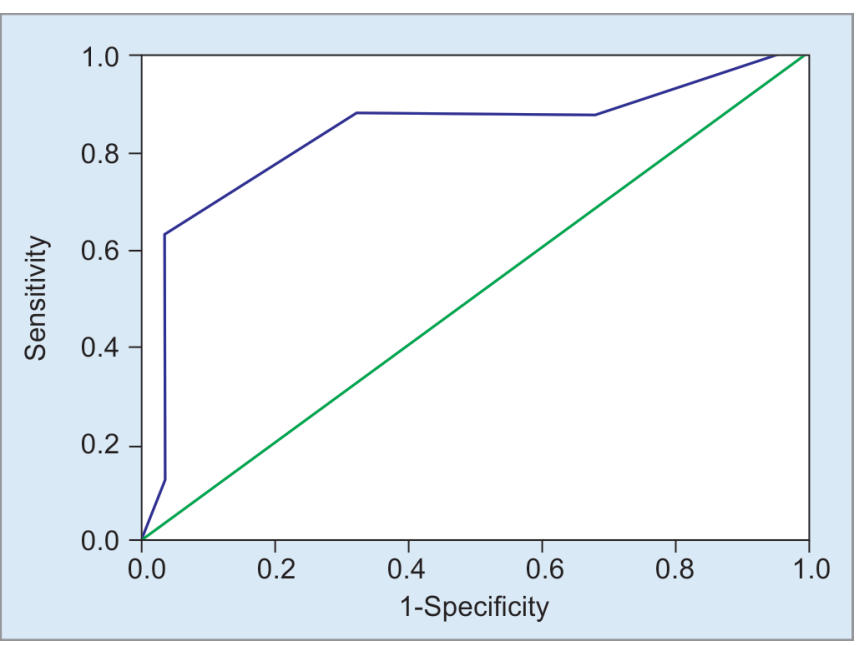

Fig. 3: ROC analysis to calculate sensitivity, specificity, NPV, and PPV of the scoring system

Table 4: Sensitivity, specificity, PPV, and NPV of each cut-off score

\begin{tabular}{llllc}
\hline Score & Sensitivity (\%) & Specificity (\%) & PPV (\%) & NPV (\%) \\
\hline 0 & 100 & 0 & 22.22 & 0 \\
1 & 100 & 3.6 & 22.86 & 100 \\
2 & 87.5 & 32.1 & 26.92 & 90 \\
3 & 87.5 & 67.9 & 43.75 & 95 \\
4 & 75 & 82.1 & 54.55 & 92 \\
5 & 62.2 & 96.4 & 83.33 & 90 \\
6 & 12.5 & 96.4 & 50 & 79.41 \\
\hline
\end{tabular}

Table 5: Mean score difference between benign and malignant

\begin{tabular}{lcl}
\hline Disease outcome & Mean score & pvalue \\
\hline Benign & $2.21 \pm 0.315$ & 0.001 \\
Malignant & $4.25 \pm 1.581$ & \\
\hline
\end{tabular}

presentation, final therapeutic management, and thereby resulting in low recorded specificity and PPV.

Benign adrenal tumors have no particular morphological highlights and most have distinguished margins all around..$^{11}$ The presence of poorly characterized margins is frequently a predictor of the more aggressive nature of ACCs. Also, ACCs are characterized by irregular, large, locally invasive heterogeneous lesions with necrosis and calcifications in up to $30 \%$ of cases on CECT. ${ }^{12}$ Nevertheless, the analysis of tumor margin, abutment, and necrosis, yielded statistically significant results to differentiate adrenal tumors in our series, and may therefore add to the interpretation of the CT in an individual case. Twenty-seven out of 28 benign tumors were recorded to have well-defined margins on $\mathrm{CT}$-thus margins on CT in our study derived the highest specificity (97.4\%) and PPV (80\%) among all the factors and reasonably high NPV (87.1\%) as well. Though almost as much as $50 \%$ of malignant adrenal masses were observed to have well-defined margins thereby resulting in decreased sensitivity. Due to certain characteristic pathological features like high mitotic activity, capsular invasion, and permeation of vascular spaces; ACCs tend to show abutment and local invasion on CT scans. In our study, abutment feature was observed not only in three-fourth of the malignant adrenal masses but also in almost one-fifth of benign lesions as well. The abutment feature was thus studied to have appreciably high NPV (91.7\%), specificity (78.6\%), and sensitivity (75\%). Malignant tumors particularly ACCs are known to have central areas of cystic necrosis and cystic degeneration. ${ }^{13}$ We recorded a high NPV (91.7\%), specificity (78.6\%), and sensitivity (75\%) for necrosis in our study. Necrosis was also found in almost one-fifth of the benign adrenal masses, almost all of them were pheochromocytoma - tumors that are also known to have necrotic areas-maybe this could explain a lower PPV of $50 \%$.

Calcifications are also among salient features of adrenal CT morphology. Being non-specific for any pathology; calcifications play an only minor role in supporting the radiological or pathological diagnosis for adrenal disorders. As far as malignant adrenal masses are concerned, irregular calcifications can be seen up to slightly less than one-third of ACCs and metastases. ${ }^{13}$ Among benign adrenal tumors, egg-shell calcification is a typical feature of an adrenal teratoma; $20 \%$ of ganglioneuroma may show punctate or discrete calcifications. Other non-specific calcifications are observed in adrenal hemangioma and pheochromocytoma. These findings reveal that calcifications are poorly associated with any specific etiopathogenesis. The same is depicted in our study with poor sensitivity and specificity. Neither did we find any significant difference in the occurrence of calcification in either disorder.

Malignant tumors like ACC are characterized with heterogeneous attenuation at unenhanced $C T$ of $>10 \mathrm{HU}$. Upon contrast enhancement $\mathrm{CT}$ scans, ACCs reflect a classical heterogeneous enhancement with a peripheral predominance. ${ }^{12-15}$ Nevertheless, derived statistically insignificant it was important to note that in our study none of the malignant tumors were observed to have homogeneous enhancement-this implies in recording highest sensitivity and NPV of $100 \%$ each. Though it was also seen as a radiological feature of $>82 \%$ of benign tumors and henceforth resulting in poor specificity and PPV. More than half tumors of our benign tumor population were pheochromocytoma, paraganglioma, and myelolipoma. Pheochromocytoma and paraganglioma are known to have classical enhancement on CT scans which may be heterogeneous due to necrotic elements. Myeloid elements in myelolipoma may show some degree of enhancement among otherwise predominant fatty tissue of specific imaging characteristics with the attenuation $<0 \mathrm{HU}$, sometimes $<$ -50 to $-70 \mathrm{HU}$ on pre-contrast CT images. ${ }^{13}$ Other benign tumor-like cysts rarely may have few heterogeneous elements on $\mathrm{CT}$ scans.

Being a retrospective study, it has its own limitations. Henceforth, we recommend further prospective studies with a large sample size for better results.

\section{ConCLUSION}

This novel scoring method including heterogeneity, margins, abutment, and necrosis play a key role in predicting adrenal malignancy accurately on CT scans.

\section{References}

1. Song JH, Chaudhry FS, Mayo-Smith WW. The incidental adrenal mass on CT: prevalence of adrenal disease in 1,049 consecutive adrenal masses in patients with no known malignancy. AJR Am J Roentgenol 2008;190(5):1163-1168. DOI: 10.2214/AJR.07.2799.

2. Lenert JT, Barnett CC, Kudelka AP, et al. Evaluation and surgical resection of adrenal masses in patients with a history of extraadrenal malignancy. Surgery 2001;130(6):1060-1067. DOI: 10.1067/ msy.2001.118369.

3. Lam KY, Lo CY. Metastatic tumours of the adrenal glands: a 30-year experience in a teaching hospital. Clin Endocri 2002;56(1):95-101. DOI: $10.1046 / \mathrm{j} .0300-0664.2001 .01435 . x$. 
4. Wang F, Liu J, Zhang R, et al. CT and MRI of adrenal gland pathologies. Quant Imaging Med Surg 2018;8(8):853-875. DOI: 10.21037/ qims.2018.09.13.

5. Gufler H, Eichner G, Grossmann A, et al. Differentiation of adrenal adenomas from metastases with unenhanced computed tomography. J Comput Assist Tomogr 2004;28(6):818-822. DOI: 10.1097/00004728-200411000-00015.

6. Yun M, Kim W, Alnafisi N, et al. 18F-FDG PET in characterizing adrenal lesions detected on CT or MRI. J Nucl Med 2001;42(12):1795-1799.

7. Grumbach MM, Biller BM, Braunstein GD, et al. Management of the clinically inapparent adrenal mass ("incidentaloma"). Ann Int Med 2003;138(5):424-429. DOI: 10.7326/0003-4819-138-5-20030304000013.

8. Mansmann G, Lau J, Balk E, et al. The clinically inapparent adrenal mass: update in diagnosis and management. Endocr Rev 2004;25(2):309-340. DOI: 10.1210/er.2002-0031.

9. Young WF. Clinical practice. The incidentally discovered adrenal mass. N Eng J Med 2007;356(6):601-610. DOI: 10.1056/NEJMcp065470.

10. Fassnacht $M$, Kreissl MC, Weismann D, et al. New targets and therapeutic approaches for endocrine malignancies. Pharmacol
Therapeut 2009;123(1):117-141. DOI: 10.1016/j.pharmthera.2009. 03.013.

11. Boland GW, Blake MA, Hahn PF, et al. Incidental adrenal lesions: principles, techniques, and algorithms for imaging characterization. Radiology 2008;249(3):756-775. DOI: 10.1148/radiol.24930 70976.

12. Fishman EK, Deutch BM, Hartman DS, et al. Primary adrenocortical carcinoma: CT evaluation with clinical correlation. Am J Roentgenol 1987;148(3):531-535. DOI: 10.2214/ajr.148.3.531.

13. Lattin GE, Sturgill ED, Tujo CA, et al. From the radiologic pathology archives: Adrenal tumors and tumor-like conditions in the adult: radiologic-pathologic correlation. Radiographics 2014;34(3):805-829. DOI: 10.1148/rg.343130127.

14. Bharwani N, Rockall AG, Sahdev A, et al. Adrenocortical carcinoma: the range of appearances on CT and MRI. AJR Am J Roentgenol 2011;196(6):W706-W714. DOI: 10.2214/AJR.10.5540.

15. Zhang HM, Perrier ND, Grubbs EG, et al. CT features and quantification of the characteristics of adrenocortical carcinomas on unenhanced and contrast-enhanced studies. Clin Radiol 2012;67(1):38-46. DOI: 10.1016/j.crad.2011.03.023. 